\title{
Gestión del diseño de producto y capacidad de aprendizaje organizativo en varios tipos de empresas del sector cerámico
}

\author{
R. CHIVA', R. LAPIEDRA', C. DEVECE², I. GIL ${ }^{2}$ \\ 'Departamento de administración de empresas y marketing, Universitat Jaume I, Av. de Vicent Sos Baynat, s/n 12071, Castellón, España \\ ${ }^{2}$ Departamento de organización de empresas, Universitat Politècnica de València, Camino de Vera s/n, 46022, Valencia, España
}

\begin{abstract}
Este trabajo estudia la relación entre la gestión del diseño de producto y la capacidad de aprendizaje organizativo en el sector cerámico español. A partir de un estudio de casos comparativo en cuatro empresas del mencionado sector, se determina qué factores facilitadores del aprendizaje organizativo son esenciales para cada una de las fases de la gestión del diseño de producto: la fase analítico conceptual y la fase técnico creativa. El estudio de casos evidencia una relación directa y positiva entre dichos factores facilitadores de aprendizaje organizativo y la gestión del diseño de producto. En concreto, cuatro de ellos están asociados a la obtención de conocimiento del mercado, la empresa y la tecnología, lo cual está vinculado a la fase analítica-conceptual del proceso de diseño de producto. Otros diez factores estarían asociados a la divulgación y uso del conocimiento, lo cual estaría vinculado a la fase técnica-creativa o la dirección integral del proceso de diseño.
\end{abstract}

Palabras clave: Gestión del diseño del producto, aprendizaje organizativo, desempeño, sector cerámico

Product design management and organizational learning capability in several company types of the ceramic sector

This study analyses the product design management in several companies of the Spanish ceramic sector and its relationship with organizational learning. We carried out a case study of four companies in order to assess the organizational learning factors involved in the two phases of the product design process: the analytical-conceptual and the technical-creative phases. The case study shows a positive relationship between the organizational learning factors analysed and the product design process. Specifically, the factors related to market, technology and organization knowledge acquisition are linked to the analytical-conceptual phase. The organizational learning factors related to knowledge dissemination and use are linked to the technical-creative phase or the global management of the design process.

Key words: Product design management, organizational learning, performance, ceramic sector

\section{INTRODUCCIÓN}

El diseño de producto es un aspecto esencial del proceso de innovación [1] y del desarrollo de nuevos productos $[2,3]$, lo cual se refleja en el grado de competitividad y desempeño empresarial. Sin embargo, parece existir una falta de comprensión de su valor y potencial $[4,5]$, lo cual se debe, según Lorenz [6], a que hasta el momento no existe una clara caracterización y sistematización del diseño del producto. Además, el proceso de diseño de producto requiere una gestión que condiciona fuertemente su eficacia $[7,8,9]$. Chea [10] considera que todavía existe una falta de consenso sobre las prácticas que requiere la gestión del diseño, hecho resaltado hace ya más de una década por Cooper y Press [11].

Aubert [1] entiende el diseño como la esencia de la innovación, el momento en el que un nuevo objeto es imaginado, materializado y formado en forma de prototipo. Así pues, el diseño está estrechamente relacionado con la innovación, ya que el propio acto de diseñar introduce siempre algo nuevo. Asimismo, dicho proceso de innovación en la empresa se concibe como un proceso de aprendizaje individual y colectivo, que responde a un patrón de búsqueda constante de nuevas ideas y resolución de problemas [12, 13], lo cual vincularía la innovación y el diseño al aprendizaje organizativo. Para diseñar es necesario aprender. Para Popadiuk and Choo [14], la innovación en la empresa depende fundamentalmente de su capacidad para organizarla como un proceso interactivo de aprendizaje. A través de este aprendizaje se crea un nuevo conocimiento tecnológico y se modifica la base del conocimiento utilizado.

Dado que la innovación y el diseño son procesos que tienen lugar en las organizaciones, las características organizativas son determinantes para su desarrollo. Por ello, averiguar qué características organizativas están implicadas en cada una de las fases del diseño es esencial. También es determinante analizar si la capacidad de aprendizaje organizativo promueve la gestión del diseño y en qué medida y aspectos [15]. Además, las fases de la gestión del diseño permitirán agrupar los factores 
facilitadores del aprendizaje organizativo entre aquellos que permiten la adquisición del conocimiento organizativo y aquellos que lo diseminan y usan [16].

El propósito de este trabajo es analizar cómo incide la capacidad de aprendizaje organizativo en la gestión del diseño de producto, tomando como marco el sector cerámico español. Se trata de comprender la capacidad de aprendizaje organizativo en los contextos y prácticas donde tiene lugar, analizando sus dimensiones o factores, además de comprender las relaciones de éstos con las actividades del diseño. De esta forma podemos sacar conclusiones sobre las características organizativas necesarias para acometer las fases de la gestión del diseño, y a su vez, podemos organizar y agrupar los factores facilitadores del aprendizaje organizativo a partir de las fases de la gestión del diseño.

Dadas las preguntas que queremos responder en este estudio y su carácter exploratorio, y las limitaciones de la literatura existente mencionadas anteriormente, entendemos que el estudio de casos es la estrategia metodológica idónea [17].

\section{DESARROLLO TEÓRICO}

\subsection{La gestión del diseño}

El diseño de producto se considera como el proceso de formalización de un producto atendiendo a requerimientos funcionales, de uso, de fabricación y de comunicación, implicando no sólo un acto creativo, sino de confluencia de aspectos técnicos, de mercado y estratégicos [18].

Los trabajos que abordan la relación entre el diseño y el desempeño de la organización ofrecen resultados similares [19]. Varios trabajos de investigación en la industria cerámica $[20,21]$ han demostrado que el diseño mejora el desempeño financiero de la empresa, incrementa su cuota de mercado y sus exportaciones. Estos trabajos mantienen que uno de los factores más importantes para la competitividad y el desempeño organizativo de una empresa es el grado de calidad de los diseños de los productos.

Las decisiones de diseño afectan no sólo a factores relacionados con el desempeño de sus productos (calidad de productos, originalidad, fiabilidad, apariencia, seguridad, facilidad de uso, durabilidad), imagen corporativa (presentación del producto, visualización o exposición, envases y embalajes, promoción), tiempo de entrega (diseño fácil de transportar) y servicio post-venta (diseño que facilita el servicio de reparación), sino también a factores vinculados al precio, tales como los costes de fabricación y de almacenamiento [22].

El concepto de gestión del diseño tiene una larga tradición y ha sido tratado tanto teóricamente $[8,11,18,23,24,25,26$ ] como empíricamente [19, 27]. La gestión del diseño puede ser entendida de diferentes formas [28], subrayando distintos aspectos o actividades e implicando variadas tipologías y acepciones. No obstante, entendemos que todas enfatizan la necesidad de ciertas actividades directivas y organizativas para optimizar el proceso de diseño. Basando nuestra definición en Gorb y Dumas [29], consideramos la gestión del diseño como una serie de actividades organizativas y directivas que son requeridas para llevar a cabo con éxito el proceso de diseño. La gestión del diseño engloba fundamentalmente dos fases: la analítico-conceptual y la técnico-creativa. En la primera de ellas, los diseñadores valoran los siguientes aspectos: el contexto socioeconómico, el concepto sociocultural del producto, los aspectos comerciales y estratégicos de la empresa, los aspectos industriales y productivos, los aspectos de distribución y logística, y los aspectos de imagen y comunicación de la empresa. Con este conocimiento se definen los atributos del producto, con los cuales se inicia la segunda fase o tarea de interpretación formal y resolución técnica para determinar el producto. Esto requiere interpretar de forma creativa las prescripciones y orientaciones aportadas en la fase anterior, así como intervenir en el resto de fases del desarrollo de producto [30].

TABLA I: RESUMEN DE LOS FACTORES QUE FACILITAN EL APRENDIZAJE ORGANIZATIVO.

\begin{tabular}{|l|c|}
\hline Experimentación, nuevas ideas, mejora continua, recompensas, apertura al cambio. & {$[34,35,36,37,38,39,40]$} \\
\hline Observación, apertura e interacción con el entorno. & {$[35,36,37,38,40]$} \\
\hline Aceptación del error y riesgo. & {$[34,35,36,37,38,40]$} \\
\hline Heterogeneidad, diversidad. & {$[35,39]$} \\
\hline Diálogo, comunicación y construcción social. & {$[36,37,38,39,40]$} \\
\hline Formación continua. & {$[34,36,38]$} \\
\hline Delegación y participación. & {$[34,40]$} \\
\hline Trabajo en equipo, importancia del grupo, espíritu colectivo, colaboración. & {$[36,37,39,40]$} \\
\hline Trabajadores con deseos de aprender. & {$[34,36]$} \\
\hline Liderazgo comprometido. & {$[37,38,40]$} \\
\hline Aprendizaje como elemento esencial de la estrategia. & {$[34,37,38]$} \\
\hline Estructura organizativa y directiva poco jerárquica y flexible. & {$[37]$} \\
\hline Conocimiento de objetivos y estrategias organizativas, accesibilidad de la información. & {$[34,38,40]$} \\
\hline Sentido del humor. & {$[39]$} \\
\hline Improvisación, creatividad. & {$[39]$} \\
\hline
\end{tabular}


Buena parte de la literatura [2, 29, 18] considera que dicho proceso de diseño de producto requiere la presencia y actuación de distintos interlocutores, no sólo de diseñadores, denominando a esta integración de interlocutores "Diseño Silencioso" [29] o "Diseño Total" [2].

\subsection{La capacidad de aprendizaje organizativo y la gestión del diseño}

El aprendizaje organizativo puede definirse como la adquisición, mantenimiento y puesta al día de conocimientos compartidos por las personas, en el que el contexto toma un papel esencial. Dicho proceso implica dos dimensiones o fases fundamentales: la adquisición del conocimiento y su diseminación y uso [16]. El concepto de capacidad de aprendizaje organizativo hace hincapié en la importancia de los factores facilitadores de aprendizaje o en la propensión organizativa a aprender. Goh y Richards [31] lo definen como las características organizativas y directivas que facilitan el aprendizaje organizativo o permiten a una organización aprender.

Tanto estudios de índole teórica [32] como de índole empírica [31,33] han sugerido cuáles pueden ser estos factores de aprendizaje. Este trabajo intenta establecer una relación de estos factores de aprendizaje con el diseño del producto. En la tabla I resumimos los principales factores subrayados por la literatura para facilitar el aprendizaje organizativo.

Parte de la literatura sobre gestión del diseño [11, 18] plantea la existencia de ciertos factores contextuales que favorecen una eficaz gestión del diseño, y que claramente se pueden vincular con los expuestos en la tabla I: estilo directivo poco jerárquico y participativo; clima de confianza y apertura; trabajo en equipo; y comunicación y diálogo frecuente entre los diseñadores y el resto de funciones. Sin embargo, estos trabajos no determinan los requisitos organizativos para cada fase del diseño, lo cual es de elevada importancia para su gestión eficaz. Los factores que facilitan el aprendizaje organizativo pueden dividirse entre los que facilitan la adquisición de conocimiento y los que promueven su diseminación y uso.

Preliminarmente podemos plantear que aquellos factores asociados a la adquisición del conocimiento estarán más vinculados a la fase analítico-conceptual. Además, aquellos factores vinculados a la utilización y divulgación del conocimiento lo estarán con la fase técnico-creativa. Este artículo pretende agrupar dichos factores y señalar cuáles son los más importantes para cada fase del diseño.

\section{EL MÉTODO DE INVESTIGACIÓN}

Dado que el propósito de nuestro estudio es exploratorio, ya que pretende profundizar en la relación entre la gestión del diseño y los factores facilitadores del aprendizaje organizativo, depurando y desarrollando teorías, el estudio de casos múltiple parece el más adecuado. Debido a que la gestión del diseño es un fenómeno más fácilmente perceptible que el aprendizaje organizativo, nuestro primer propósito era determinar a priori distintas situaciones sobre la gestión del diseño de producto, para luego estudiar su vinculación con el aprendizaje organizativo. Por lo tanto, elegimos la gestión del diseño de producto como concepto discriminante. De acuerdo con la lógica de la réplica, debemos tratar de encontrar empresas que gestionen eficazmente el diseño y empresas que no lo hicieran. En esta tarea fue fundamental la colaboración con el Instituto Tecnológico Español del Diseño Cerámico, ALICER (ITC).

El conocimiento sobre el diseño y su gestión en el sector cerámico por parte de los técnicos de ALICER permitió seleccionar distintas empresas que, a priori, gestionaban adecuadamente el diseño y a otras que no lo hacían. Para esta dicotomización, los expertos se basaron en aquellas actividades implicadas en la gestión del diseño de las cuales tenían información. Las características que permitieron clasificar a las empresas fueron: situación del diseño en la estructura organizativa, grado de importancia del diseño en la empresa, y grado de Diseño Silencioso" o Diseño Total" en la empresa. Basándonos en la literatura previa se consideró que un elevado grado de las dos últimas características implicaba una eficaz gestión del diseño. Por el contrario, la primera de estas características, simplificada a la existencia o no de departamento de diseño, se considera como indeterminada por la literatura por no haber una validación empírica sólida. Así pues, se dividió la muestra en dos grupos de empresas: aquellas que mostraban un grado elevado de dicho par de características (importancia del diseño en la empresa, y grado de "Diseño Total") y aquellas que no lo hacían. Por otro lado, y con objeto de enriquecer el estudio, se buscó que dentro de la réplica literal de cada grupo existieran empresas que poseyeran departamento de diseño y otras que no. Posteriormente se discriminó las empresas por su accesibilidad y potencial interés. Con el objeto de incentivar a las empresas seleccionadas para que accedieran al estudio y que, además, al final contrastaran y verificaran nuestros resultados, se les prometió la entrega de una auditoría de gestión de diseño, junto a un análisis de la capacidad de aprendizaje organizativo. Finalmente, cuatro accedieron a nuestro estudio. Dos de ellas gestionaban a priori eficazmente el diseño, de las cuales una tenía departamento de diseño y otra no. Las otras dos no gestionaban eficazmente el diseño, de las cuales también una tenía departamento de diseño y otra no.

Las cuatro empresas seleccionadas forman parte de la industria española productora de pavimentos y revestimientos cerámicos, el cual tiene como característica más destacable su alto grado de concentración geográfica, ya que más del $80 \%$ de las empresas se encuentran ubicadas en la provincia de Castellón. Casi el 90\% de la producción nacional tiene origen en esta provincia, en la cual están también se concentran sus proveedores o empresas vinculadas como fabricantes de maquinaria; fabricantes de fritas, esmaltes y colores cerámicos; fabricantes de piezas especiales; gabinetes de diseño; fabricantes de moldes, etc.

\subsection{Métodos de obtención de datos}

Los métodos de obtención de la información en nuestro estudio de casos fueron las entrevistas estructuradas, los documentos y archivos, y la observación directa. Cada uno de ellos fue utilizado para analizar los distintos conceptos y ámbitos de la empresa: aprendizaje organizativo, gestión del diseño de producto e información general sobre la empresa. La recogida de datos fue llevada a cabo por parte de los autores, con la ayuda de dos técnicos de ALICER. 
Aprendizaje organizativo. Para el desarrollo de la entrevista estructurada nos basamos en las distintas características que facilitaban la existencia de aprendizaje organizativo recopiladas y resumidas en la Tabla II, y en dos cuestionarios que evalúan el contexto organizativo y directivo que favorece la existencia de aprendizaje organizativo, concretamente "Organizational Learning Survey" de Goh y Richards [31], y "Learning Environment Survey" de Tannenbaum [34]. La estructura de la entrevista final constaba de 70 preguntas, que podían dar pie, en algunos casos, a ejemplos o respuestas largas. Con anterioridad a la realización del trabajo de campo en las empresas seleccionadas se llevó a cabo un pre-test sobre técnicos de ALICER con objeto de probar y validar las preguntas. A partir de este pre-test se mejoraron algunas preguntas, y se eliminaron y se añadieron otras.

Las entrevistas estructuradas en las empresas seleccionadas se realizaron a todas aquellas personas vinculadas de alguna forma al proceso de gestión y diseño de producto, siendo éstos responsables y miembros de departamentos o áreas como diseño, gerencia, producción, I+D, marketing y comercial, finanzas y recursos humanos. Si bien las preguntas iban encaminadas a descubrir el grado de existencia de determinadas características contextuales, la actividad elegida sobre la cual se plantearon estas preguntas era el proceso y gestión del diseño de producto (Ejemplo: ¿Cómo es la comunicación en el proceso de diseño de producto?, ¿Constante, frecuente?, ¿Puede ofrecer ejemplos?). Se realizaron al menos 15 entrevistas por empresa, cada una de las cuales tuvo una duración aproximada de dos horas, aunque algunas llegaron a durar casi tres.

Gestión del diseño de producto. En relación con este concepto, revisamos los distintos modelos de auditorías de diseño, seleccionando el de la Sociedad Española para el Desarrollo del Diseño y la Innovación [42] por considerar que tiene una perspectiva considerablemente ecléctica. Además, este modelo fue adaptado ligeramente para hacer especial énfasis en la gestión del diseño de producto y en la idiosincrasia del sector cerámico español. Las dos personas entrevistadas fueron el Director General y el responsable del diseño de producto de la empresa, a los cuales se les preguntó sobre los siguientes aspectos: el departamento de diseño, el proceso de diseño, desarrollo y comunicación de producto, fuentes de diseño, características de los proyectos de diseño, grado de implicación de las personas vinculadas al diseño, y papel e importancia del diseño en la empresa. La duración de la entrevista osciló entre 90 y 120 minutos.

Teniendo en cuenta la metodología del estudio de casos y la importancia implícita del contexto organizativo, se recogió también información sobre las características generales de cada una de las empresas. Para ello, se realizó una entrevista con Gerencia sobre las competencias distintivas de la empresa, factores críticos de éxito, aspectos relativos a su negocio, clientes, producción, tecnología y estructura organizativa.

\section{RESULTADOS}

A continuación resumimos la información general de cada una de las empresas, recogida principalmente a través de entrevistas al Director General y a los responsables de cada una de las áreas funcionales de la empresa. La tabla III resume las principales características de estas empresas. Los nombres de las empresas son alterados para asegurar la confidencialidad.

Empresa "Líder en tecnología". Es la única empresa no PYME y no familiar de las cuatro, aunque sus propietarios son a su vez familias que poseen otras empresas en este sector. Dado su tamaño, están comparativamente muy burocratizados y departamentalizados, destacando la existencia de los departamentos de marketing, calidad o recursos humanos. Sus prioridades o criterios de éxito están basados en la innovación o mejora tecnológica y la calidad, cualidades que se utilizan para mostrar al distribuidor que venderá sin dificultad sus productos. Hacen un importante hincapié en la imagen corporativa. Por otro lado, muestra elevados costes de fabricación y almacenamiento, debido a su enorme gama de productos y a su inversión tecnológica.

Empresa "Seguidor". Empresa familiar con limitadas programaciones estratégicas, reflejado en un planteamiento más bien seguidor. Se centran comparativamente más en el proceso productivo. Mantienen una pobre política de marketing y elevados costes de fabricación y almacenamiento originados por una necesidad de ofrecer todo tipo de productos y en cualquier cantidad.

TABLA II: RESUMEN DE LAS CARACTERÍSTICAS GENERALES DE LA EMPRESA.

\begin{tabular}{|l|c|c|c|c|}
\hline CARACTERístiCA & “Líder en tecnología" & “Seguidor” & “Joven” & “Observador de mercado" \\
\hline Año fundación & 1967 & 1979 & 1994 & 1967 \\
\hline Número de trabajadores & 750 & 150 & 103 & 40 \\
\hline Importancia del diseño en la empresa & No & No & Sí & Sí \\
\hline Diseño silencioso o total & Apenas & Apenas & Sí & No \\
\hline Departamento de Diseño & Sí & No & Sí & No \\
\hline
\end{tabular}


Empresa "Joven". Joven empresa fundada por una persona con elevada experiencia en el sector que había abandonado una empresa familiar de cual era miembro. Sus hijos, todos con formación universitaria, son parte importante del equipo directivo. Consideran tan importante la innovación tecnológica como el diseño y el marketing, aunque reconocen su estadio inicial en todos esos aspectos. A pesar de ostentar elevados costes de fabricación y almacenamiento, se centraran en disminución cuando su producto se diferencie de sus competidores por tecnología y diseño.

Empresa "Observador de mercado". Empresa familiar, dirigida conjuntamente por un miembro de la familia y un directivo profesional muy orientado al diseño y al marketing, y con experiencia en otros sectores. Su negocio demuestra una aproximación más amplia, al ofrecer pavimentos y revestimientos de otros materiales, no sólo cerámicos. Se definen como innovadores de producto, basado en un importante énfasis en el diseño, ya que la estrategia de la empresa gira a su alrededor. Sin embargo, no dejan de lado la innovación tecnológica. Otras características que la definen son: la realización de pequeñas investigaciones de mercado y de tendencias de diseño para definir producto, la posesión de una amplia red de comercialización consolidada, elección de distribuidores exclusivos por zonas o áreas comerciales, y una excelente previsión de ventas logrando apenas productos almacenados. Consideran que la rentabilidad de sus productos es elevada por su mayor valor añadido y un ciclo de vida más prolongado, ya que sus distribuidores aceptan sus precios y sus plazos de entrega.

\subsection{La capacidad de aprendizaje organizativo}

En la tabla IV queda reflejada la situación de las cuatro empresas con respecto a las características que facilitan el aprendizaje organizativo. La idea central que se extrae es que las empresas "Joven" y "Observador de mercado" demuestran poseer en mayor grado estas características que las empresas "Líder en tecnología" y "Seguidor". Esto implicaría que estas últimas organizaciones tendrían una menor capacidad de aprendizaje organizativo, especialmente en lo que respecta a la gestión del diseño de producto, ya que la mayoría de las preguntas en las entrevistas giran entorno a esa actividad. Además, observamos que "Observador de mercado" muestra en ciertas características una mayor capacidad de aprendizaje que "Joven". Estas características son: una mayor apertura y capacidad de cambio innovador, una cultura que favorece la aportación de más ideas críticas y una máxima atención al entorno.

TABLA III: SITUACIÓN DE LOS FACTORES QUE FACILITAN EL APRENDIZAJE ORGANIZATIVO EN LAS EMPRESAS EN RELACIÓN CON LA ACTIVIDAD DE GESTIÓN DEL DISEÑO DE PRODUCTO

\begin{tabular}{|c|c|c|c|c|}
\hline & "Líder en tecnología" & "Seguidor" & "Joven" & $\begin{array}{l}\text { "Observador } \\
\text { de mercado" }\end{array}$ \\
\hline Experimentación en diseño & Bajo & Bajo & Alto & Muy alto \\
\hline Se alienta la crítica & Bajo & Bajo & Moderado & Alto \\
\hline Facilidad al cambio & Bajo. & Bajo & Alto & Muy alto \\
\hline Atención al entorno & Moderado & Muy bajo & Moderado & Muy alto \\
\hline Aceptación del riesgo & No & No & Sí & Sí \\
\hline Heterogeneidad, diversidad (en diseño) & Moderado & Moderado & Alto & Alto \\
\hline Comunicación Formal & Moderada & Baja & Alta & Alta \\
\hline Comunicación informal & Baja. & Moderada & Alta & Alta. \\
\hline Formación continua & Sí & Sí & Sí & Sí \\
\hline Delegación & Baja & Muy baja & Muy alta & Muy alta \\
\hline Participación en la toma de decisiones. & No & No & Media & Media \\
\hline Trabajo en equipo & Rara vez & Nunca & Siempre & Con frecuencia \\
\hline Rivalidades entre departamentos. & Alta & Moderada & Muy baja & Muy baja \\
\hline Trabajadores con deseos de aprender & Sí. & Sí. & Sí. & Sí. \\
\hline Liderazgo comprometido (con el diseño) & No & No & Sí & Sí \\
\hline Aprendizaje como elemento esencial de la estrategia & No & No & No & No \\
\hline Distancia jerárquica & Sí. & Sí. & No. & No. \\
\hline Transmisión de conocimiento entre áreas & Muy bajo & Muy bajo & Muy alto & Alto. \\
\hline Secretos respecto al plan estratégico & Muy alto & Muy alto & Bajo & Bajo \\
\hline Conocimiento de propósito y objetivos de la empresa & Muy alto. & Muy alto & Muy alto & Alto \\
\hline Sentido del humor & Apenas. & Medio. & Sí. & Sí. \\
\hline Improvisación, creatividad & Baja & Baja & Alta & Alta \\
\hline
\end{tabular}




\subsection{Gestión del diseño de Producto}

En relación con el diseño del producto, las conclusiones de los resultados fueron las siguientes: Primero, las empresas "Joven" y "Observador de mercado" gestionan el diseño más eficazmente que "Líder en tecnología" y "Seguidor", dado que muestran un mayor número de actividades consideradas como requisitos para una gestión eficaz. Segundo, “Observador de mercado" demuestra un planteamiento más completo al realizar y hacer hincapié en la planificación del diseño de producto, lo que implica la importancia del conocimiento de la empresa, su mercado y la tecnología. Tercero, la existencia del departamento de diseño no parece necesaria para gestionar el diseño eficazmente, puesto que "Líder en tecnología" y "Joven" poseen departamento de diseño, mientras que "Seguidor" y "Observador de mercado" no. Cuarto, si analizamos las fuentes del diseño, la utilización del diseño gratuito ofrecido por los fabricantes de esmaltes, el papel predominante de los comerciales en el diseño, un papel del diseño limitado a la realización de bocetos parecen repercutir negativamente a la gestión del diseño del producto.

De la misma manera, una escasa implicación de otras áreas en el proceso de diseño, y un entendimiento del diseño como instrumento para ofrecer productos similares a los que están teniendo éxito, está asociado a una ineficaz gestión del diseño de producto. Así, una posición dominante de los comerciales en cuestiones de diseño se ve como negativa, mientras que su participación e integración como un miembro más en las decisiones sobre diseño es positiva. De la misma manera, una excesiva preocupación y adaptación a los deseos del cliente es negativa, mientras que la utilización del conocimiento de los gustos del cliente como guía para la diferenciación y la incorporación de nuevos materiales y mejora de la calidad es positiva. El mejor resultado se da cuando se realiza una eficaz y completa planificación del diseño, basándose en las prioridades de la empresa, combinado con el análisis de futuras tendencias de producto e investigaciones de mercado, y se hace hincapié en la planificación del diseño.

\subsection{Relación entre la capacidad de aprendizaje organizativo y la gestión del diseño de producto}

Dadas las diferencias entre la pareja de empresas "Líder en tecnología" y "Seguidor" por un lado, y "Joven" y “Observador de mercado" por otro, tanto en lo referente a aprendizaje organizativo como a gestión del diseño de producto, tratamos de establecer vínculos entre las características que facilitaban el aprendizaje organizativo que se daban en la segunda pareja. Así, respecto a las características para una eficaz gestión del diseño de producto, las empresas "Joven" y "Observador de mercado" destacan por:

- Implicación del diseño en otros aspectos del desarrollo de producto (Papel amplio de la función de diseño)

- Elevada implicación de otros departamentos o áreas en el proceso de diseño ("Diseño Silencioso" o "Diseño Total").

- El papel relevante y equilibrado de diseñadores o gestores de diseño con respecto al resto de áreas y funciones.

- Una elevada importancia del diseño de producto (fuentes de diseño con elevado valor añadido).

- Planificación del diseño: se tienen en cuenta las prioridades de la empresa y sus recursos.
La pareja de empresas "Joven" y "Observador de mercado" también destaca en características que facilitan el aprendizaje organizativo ya comentadas, como aceptación del riesgo, heterogeneidad y diversidad (en la toma de decisiones del diseño), comunicación interna constante, delegación y participación (dirección por objetivos), trabajo en equipo e inexistencia de rivalidades, liderazgo comprometido, poca distancia jerárquica, transferencia de conocimiento (ausencia de obsesión por los secretos), comprensión y conocimiento de la empresa y sus prioridades, sentido del humor y creatividad.

También es importante destacarciertas características únicas de "Observador de mercado" en lo referente a ambos aspectos. Esta empresa destaca sobre "Joven" por su hincapié en la planificación del diseño, y en su característica diferencial: la importancia del conocimiento de la empresa, de su mercado y de la tecnología, lo cual se relaciona con sus características diferenciales citadas en el apartado de aprendizaje organizativo. El resto de características comunes a estas dos empresas se vinculan a la otra fase del diseño de producto: la fase técnico-creativa.

\section{CONCLUSIONES}

La figura 1, el modelo teórico integrador, resume los resultados del estudio de las relaciones entre la gestión del diseño y los factores que facilitan el aprendizaje organizativo.

Uno de los objetivos de este trabajo era establecer cómo los factores que facilitan el aprendizaje organizativo inciden sobre la gestión del diseño. El estudio de casos evidencia una relación directa y positiva entre dichos factores y la gestión del diseño de producto. En concreto, cuatro de ellos (ver figura 1) están asociados a la obtención de conocimiento del mercado, la empresa y la tecnología, lo cual está vinculado a la fase analítica-conceptual del proceso de diseño de producto.
Facilitadores del Aprendizaje Organizativo
Gestión del diseño del producto

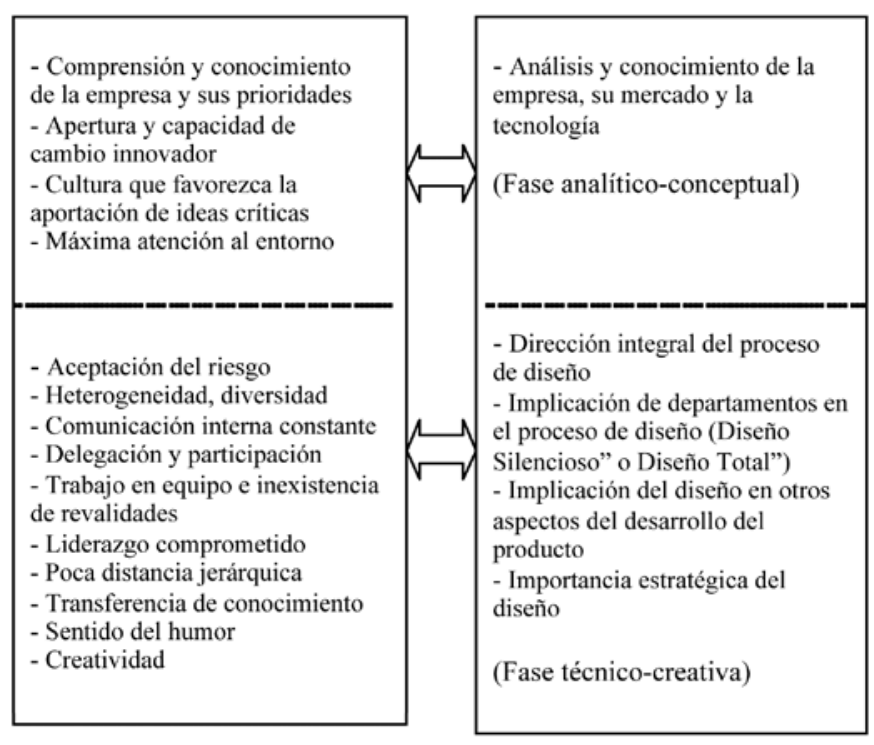

Figura 1: El modelo teórico integrador de los Factores que facilitan el Aprendizaje Organizativo y la Gestión del Diseño de Producto. 
Además de las características exclusivas de "Observador de mercado", que hacían especial hincapié en la planificación del diseño, añadimos la comprensión y el conocimiento de la empresa y de sus prioridades. Esta incorporación del conocimiento de la empresa está justificada porque ambas empresas ("Joven" y "Observador de mercado") lo tenían en cuenta en dicha fase previa del diseño.

Las otras diez características estarían asociadas a la divulgación y uso del conocimiento, lo cual estaría vinculado a la fase técnica-creativa o la dirección integral del proceso de diseño. Dicha dirección integral consistiría en: una implicación del diseño en otros aspectos del desarrollo de producto como producción, I+D, marketing, comunicación etc.; una elevada implicación de otras áreas para llevar a cabo el proceso de diseño de producto ("Diseño Silencioso" o "Diseño Total"); y por último, una importancia estratégica del diseño reflejada en su papel a la hora de tomar decisiones de producto, su lugar en la estructura organizativa, las fuentes de diseño utilizadas y los recursos recibidos.

Las siguientes proposiciones resumen las relaciones planteadas anteriormente y mostradas en la figura 2.

- Proposición 1: Los factores esenciales que facilitan el aprendizaje organizativo en el contexto de la gestión del diseño de producto son: comprensión y conocimiento de la empresa y sus prioridades, apertura y capacidad de cambio innovador, cultura que favorezca la aportación de ideas críticas y máxima atención al entorno, aceptación del riesgo, heterogeneidad y diversidad, comunicación interna constante, delegación y participación, trabajo en equipo e inexistencia de rivalidades, liderazgo comprometido, poca distancia jerárquica, transferencia de conocimiento, sentido del humor y creatividad.

- Proposición 2: Los cuatro primeros factores (ver tabla I) asociados a la adquisición de conocimiento, están relacionados con la actividad de la gestión del diseño vinculada a la fase analítico-conceptual: el conocimiento de la empresa, el mercado y la tecnología.

- Proposición 3: Los diez últimos factores (ver tabla I) asociados a la divulgación y uso de conocimiento, están relacionados con la actividad de la gestión del diseño vinculada a la fase técnico-creativa: la dirección integral del proceso de diseño.

Este estudio de casos nos ha permitido clasificar o agrupar los factores facilitadores o la capacidad de aprendizaje organizativo en dos niveles: el primero vinculado a la obtención de conocimiento; y el segundo a su divulgación y uso. Además, a partir de esta investigación hemos mejorado nuestro conocimiento de la gestión del diseño de producto en cuanto a sus actividades y a los factores que facilitan su eficacia desde la capacidad de aprendizaje organizativo.

Sin embargo, deben ser reconocidas algunas limitaciones, además de las propias de los estudios de casos. Primero, la investigación empírica fue realizada en el sector cerámico español, cuyas características idiosincrásicas podrían haber afectado a los resultados del estudio, por lo que su generalización a otras empresas manufactureras debe hacerse con precaución. Segundo, la valoración del desempeño se efectúa sobre un ámbito temporal corto, lo cual impide considerar a ciencia cierta los resultados directos de la gestión del diseño de producto.

Finalmente, ofrecemos algunas sugerencias de futuras investigaciones que complementarían este estudio, superando algunas de sus limitaciones. Primero, corroborar y perfeccionar las proposiciones planteadas a través de estudios cuantitativos y estudios similares en otros sectores y países. Segundo, realizar un estudio similar con otra actividad organizativa como la gestión de la I+D o de la calidad, con el objeto de analizar hasta qué punto estos factores que facilitan el aprendizaje organizativo son dependientes del tipo de actividad, y en el caso de serlo, cuáles son sus diferencias.

\section{BIBLIOGRAFÍA}

(1) J. E. Aubert, "Innovation in small and medium firms" OCDE, París 1982.

(2) S. Jenkins, S. Forbes and T. S. Durrani, Managing the product development process (part 1, an assessment), Int. J. Environ. Technol. Manage, 13, 359378 (1997).

(3) P. Trott, "Innovation Management and New Product Development" Prentice Hall, NY 2008.

(4) S. Potter, R. Roy, C. Capon, M. Bruce, V. Walsh and J. Lewis, "The benefits an costs of investment in design: using professional design expertise in Product, engineering and graphic projects" Design Innovation Group, Manchester 1991.

(5) F. J. H. M. Verhees and M. T. G. Meulenberg, Market Orientation, Innovativeness, Product Innovation, and Performance in Small Firms, J Small Bus manage, 42, 134-154 (2004).

(6) C. Lorenz, Harnessing design as a strategic resource, Long Range Plann, 27, 73-84 (1995)

(7) R. Chiva and J. Alegre, Investment in design and firm performance: the mediating role of design management, J Prod innovat Manag, 26, 424-440 (2009).

(8) M. Bruce and B. Morris, Managing external design professionals in the product development process, Technovation, 14, 585-600 (1994).

(9) M. Rungtusanatham and C. Forza, Coordinating Product Design, Process Design, and Supply Chain Design Decisions Part A: Topic Motivation, Performance Implications, and Article Review Process, J Oper Manag, 23, 257-65 (2005).

(10) A. C. Chea, Exemplary Models of Firm Innovation: Strategy and Leadership for the Twenty-First Century Competitive Environment, Int Bus Res, 2, 9-15 (2009).

(11) R. Cooper and M. Press, "The design agenda" John Wiley and sons, Chichester, UK 1995.

(12) D. E. Klingner and G. M. Sabet, Knowledge management, organizational learning, innovation and technology transfer, Comparative Technology, Transfer and Society, 3, 199-210 (2005).

(13) E. Nevis, A. J. DiBella and J. M. Gould, Understanding organization learning systems, Sloan Manage Rev, 36, 73-85 (1995).

(14) S. Popadiuk, C. W. Choo, Innovation and Knowledge Creation: How are these Concepts Related?, Int J Inform Manage, 26, 302-12 (2006).

(15) R. Chiva, J. Alegre and R. Lapiedra, Measuring organizational learning capability among the workforce, Int J Manpower, 28, 224-242 (2007).

(16) G. P. Huber, Organizational learning: the contributing processes and the literatures, Organ Sci, 2, 88-115 (1991).

(17) R. K. Yin, "Case study research: design and methods" Sage Publications, London, 1989.

(18) V. Walsh, Design, innovation and the boundaries of the firm, Res Policy, 25, 509-29 (1996).

(19) R. Roy and S. Potter, The commercial impacts of investment in design, Des Stud, 14, 171-193 (1993).

(20) M. Press, "Design Management in the ceramics industry" Staffordshire University design research centre, Stoke on Trent, 1991.

(21) J. Alegre-Vidal, R. Lapiedra-Alcamí and R. Chiva-Gómez, Linking operations strategy and product innovation: an empirical study of Spanish ceramic tile producers, Res Policy, 33, 829-839 (2004).

(22) B. Von Stamm, "Managing Innovation, Design and Creativity"Wiley \& Sons, Chichester, UK, 2003.

(23) R. W. Veryzer and S. R. Habsburg, Managing the challenge of Design for Innovation, Des Manage J, 10, 29-34 (1999).

(24) F. Joziasse, Corporate Strategy: Bringing Design Management into the Fold, Des Manage J, 11, 36-41 (2000).

(25) B. H. Jevnaker, How Design Becomes Strategic, Des Manage J, 11, 41-47 (2000). 
(26) E. M. Olson, S. F. Slater and R. D. Cooper, Managing Design for Competitive Advantage: A process approach, Des Manage J, 11, 10-17 (2000).

(27) R. Roy and J. C. Riedel, Design and Innovation in successful produc competition, Technovation, 17, 537-549 (1997).

(28) M. Bruce and J. R. Bessant, "Design in Business" Prentice Hall, Essex, 2002.

(29) P. Gorb and A. Dumas, Silent design, Des Stud, 8, 150-156 (1987).

(30) J. M. Iváñez, "La gestión del diseño en la empresa", McGraw-Hill Management, Madrid, 2000

(31) S. Goh and G. Richards, Benchmarking the learning capability of organisations, Eur Manage J, 15, 575-83 (1997).

(32) K. E. Weick and F. Westley, Organizational learning: affirming an oxymoron, in S. R. Clegg, C. Hardy and W.R. Nord, eds., "Handbook of organizational studies" 440-458, Sage, London, 1996.

(33) S. Gherardi, D. Nicolini and F. Odella, Toward a social understanding of how people learn in organizations, Manage Learn, 29, 273-97 (1998).

(34) S. I. Tannenbaum, Enhancing continuous learning: diagnostic findings from multiple companies, Hum Resource Manage, 36, 437-52 (1997).

(35) B. Hedberg, How Organizations Learn and Unlearn, in P. Nystrom and W. H. Starbuck, eds., "Handbook of Organizational Design" 1, 3-27, Cambridge University Press, London, 1981.
(36) D. Leonard-Barton, The factory as a learning laboratory, Sloan Manage Rev, $34,23-38$ (1992).

(37) D. Ulrich, T. Jick and M. A. Von Glinow, High-Impact Learning: Building and Diffusing Learning Capability. Organ Dyn, 22, 52-66 (1993).

(38) E. C. Nevis, A. Dibella, and J. M. Gould, Understanding organizations as learning systems, Sloan Manage Revi, 36, 73-85 (1995).

(39) S. C. Goh and G. Richards, Benchmarking the learning capability of organizations, Eur Manage J, 15, 575-83 (1997).

(40) K. E. Weick and F. Westley, Organizational learning: affirming an oxymoron, in S. R. Clegg, C. Hardy and W. R. Nord, eds., "Handbook of Organization Studies" 440-458,Sage: London (1996).

(41) J. S. Brown and P. Duguid, Enacting Design for the Workplace, in P.S. Adler and T. A. Winograd, eds., "Usability: Turning Technologies into Tools" 41-65, Oxford University Press, New York, 1992.

(42) DDI, "Auditoría de diseño", DDI (Sociedad Estatal para el Desarrollo del Diseño y la Innovación), Madrid, 1992.

Recibido: 28/02/2011

Aceptado: $16 / 05 / 2012$ 